\title{
The Architecture of Time, Part 3: Project Management in Two-Dimensional Time
}

\author{
Thomas Gangale ${ }^{1}$ \\ OPS-Alaska, Petaluma, California, 94952
}

\begin{abstract}
The Gantt chart depicts the current prediction of how the future is going to unfold, but it does not adequately tell the history of the project's efforts to get to that future. This is because the Gantt chart is really a series of charts, each of which has only one dimensiontime-running from left to right across the page. The vertical dimension does not signify a continuum, but is simply a means of compiling the schedule of individual albeit interdependent milestones onto one visual display. It is today's snapshot of how those milestones relate to each other in the overall schedule, but it does not show very well how those milestones have influenced each other over time.
\end{abstract}

It would be valuable to have some way of displaying all of yesterday's schedule snapshots as well as today's on a single chart. Then one could see at a glance the nature of the trends for each milestone and how they feed into the trend of the master schedule. This can be done by reformatting the chart so that a second dimension of time is displayed. The time axis on a conventional schedule chart isn't real time in the sense of showing the occurrence of actual events with respect to the passage of time; it is only projected time- the pseudo-temporal axis along which a manager positions his best guesses of when he will achieve his milestones. In a chart that not only contains the pseudo-temporal axis but also a second axis corresponding to the actual passage of time, one can see the history of a program's perception of the future - how schedules have been influenced by the course of events, and how they have evolved with time. Presentation of schedules in this new two-dimensional format provides managers an extra dimension of information, enabling them to make better subjective judgments on how realistic the current schedule is, and how it may change in the face of possible future events.

\section{History of Ideas}

$\mathrm{T}$ he two best known project management charts are the Gantt and PERT. Henry Gantt published his chart design in 1910 (Gantt). An online source claims that a similar idea was developed by Karol Adamiecki, under the name Harmonogram; however Adamiecki did not publish his work until 1931. Gantt charts have become a common technique for representing the phases and activities of a project work breakdown structure (WBS), so they can be understood by a wide audience.

The Program Evaluation and Review Technique (PERT) is a model for project management invented by Booz Allen Hamilton, Inc. under contract to the United States Department of Defense's US Navy Special Projects Office in the 1950s as part of the Polaris submarine-launched ballistic missile project. PERT is a method to analyze the tasks involved in completing a given project, especially the time needed to complete each task, and identifying the minimum time needed to complete the total project. At about the same time, the DuPont Corporation developed the critical path method.

Much less known is the slip/progress chart or trend chart, which presents two dimensions of time. The passage of historical time (the past) is measured along one axis, and at discrete points in historical time, events in a project's schedule (the future) are projected along the orthogonal axis. The slip chart is mentioned in the literature as early as 1973 (Brooke). It also appears in the appendix of the official NASA history of the Ranger lunar probe project (Hall 1977); however, nowhere in the text is the chart referenced, either as a tool for program management or for historical analysis.

\footnotetext{
${ }^{1}$ Executive Director, AIAA Professional Member.
} 


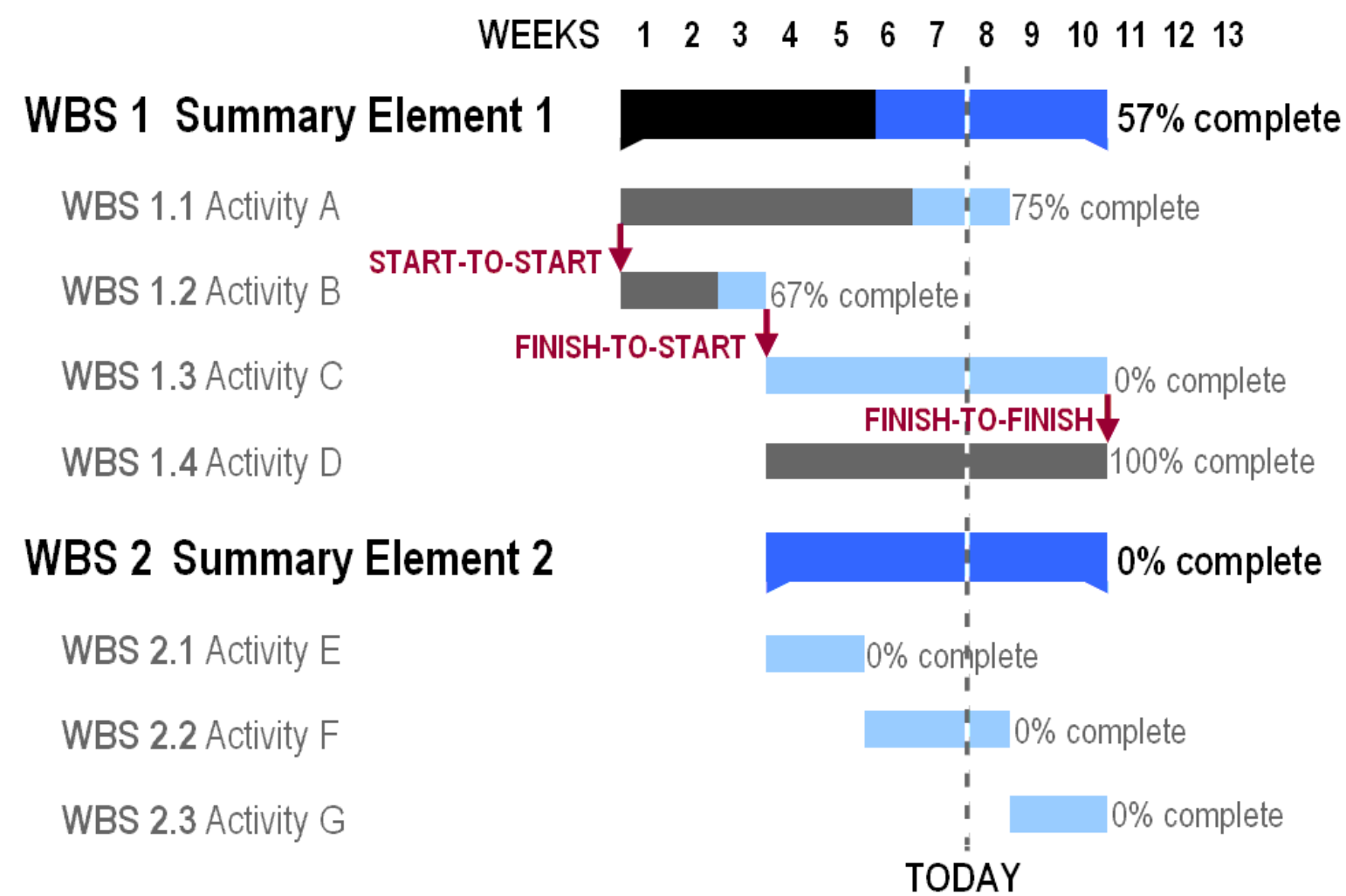

Figure 1. Gantt Chart

Source: http://en.wikipedia.org/wiki/Gantt_chart

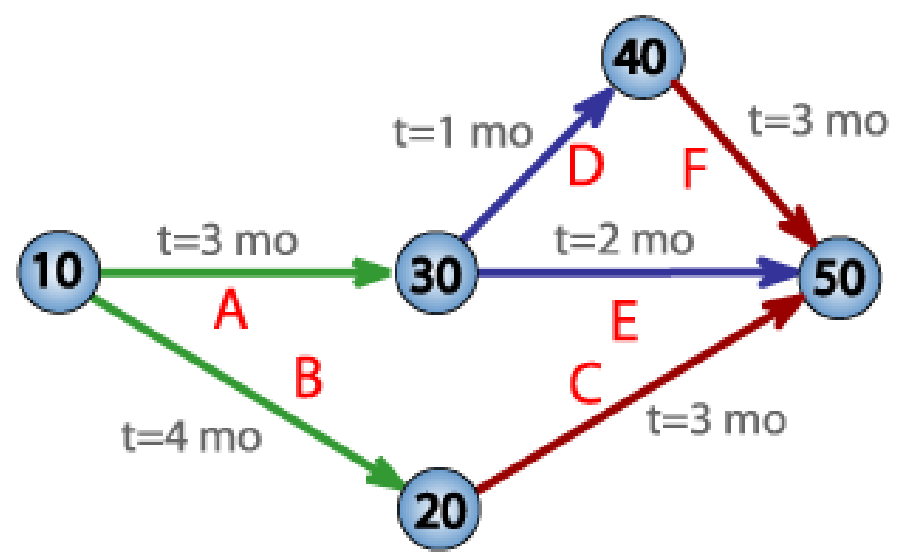

Figure 2. PERT Chart

Source: http://en.wikipedia.org/wiki/Pert_chart

\section{Time Maps}

As it happens, I independently developed the same concept at about the same time (late 1970s). At the time, my chief focus was historical analysis. In 1975, I became interested in researching manned space missions that NASA had canceled, and over the next few years, I developed quite a list of them. Additionally, there were the canceled US Air Force programs Dyna Soar and Manned Orbiting Laboratory. I began to see that simply listing these "lost missions of the Space Age" in some logical sequence, along with the missions that actually flew was problematic. As I attempted to list all of these missions chronologically, the question for canceled missions, or for missions that 
were proposed but not funded, when were they scheduled when? Put another way, the question was not only their scheduled launch dates, but the dates of those schedules. I needed more than a simple linear chronological list; I concluded that I needed to portray time in two dimensions, past and future. With this schema, I could portray a chronological topography of a program's development: a time map.

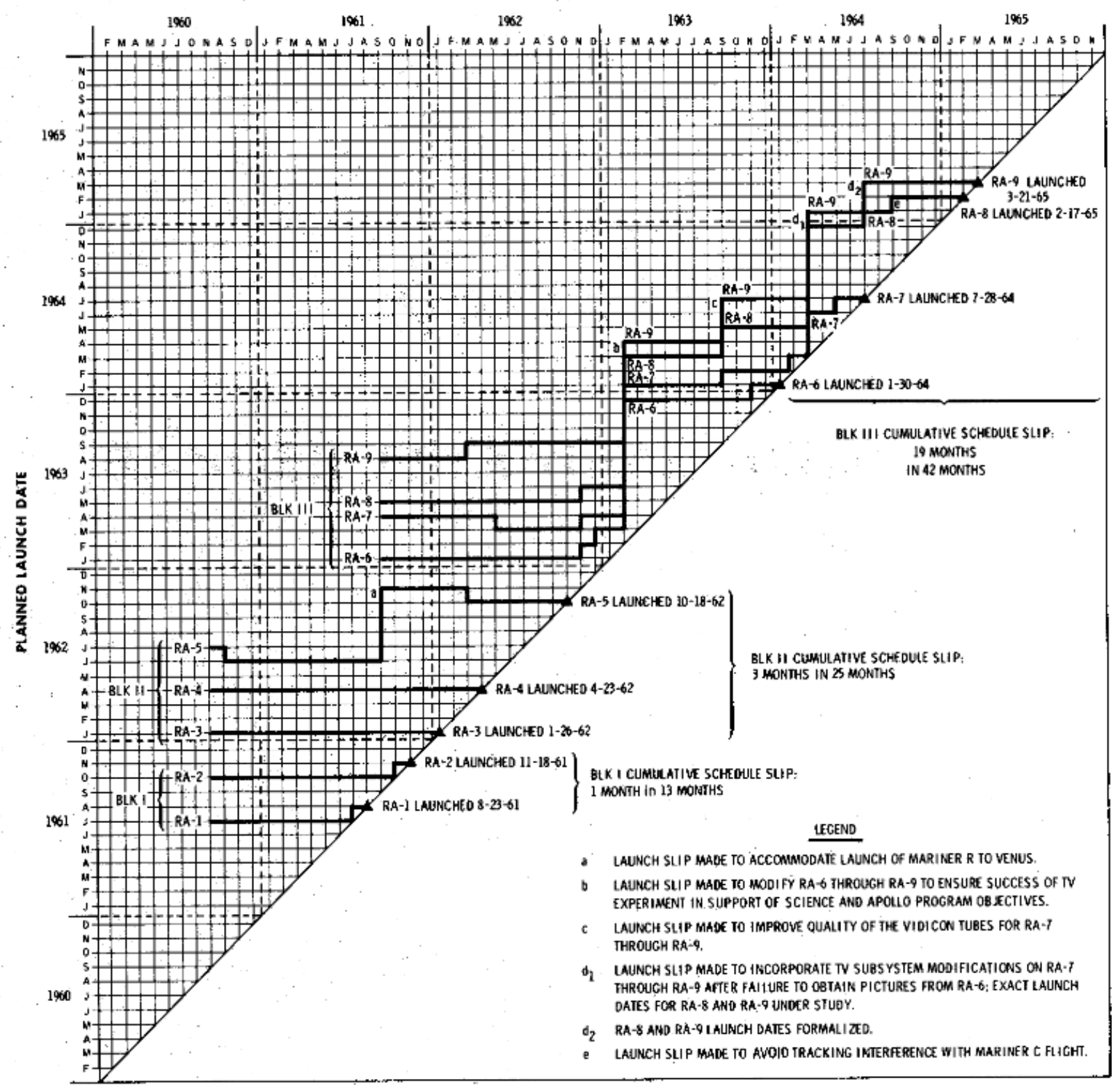

Figure 3. NASA Slip Chart

Source: http://history.nasa.gov/SP-4210/images/gif/one_third/appf_1.gif

Figures 4 and 5 are typical time maps. It can be seen that Gordon Cooper's Mercury-Atlas 9 mission, launched in May 1963, began as one of four proposed 18-revolution (1.5 day) missions, whereas the original purpose of Project Mercury was limited to recovering astronauts after three revolutions. Extending the capability of the Mercury spacecraft to 1.5 days required some redesign. At about the same time these four extended Mercury missions were proposed in July 1961, the first launch schedule for the Mercury Mark II (later renamed Gemini) program was developed. Since the schedule for the extended Mercury missions fell on top of the early Gemini schedule, there must have been some question as to their utility, first because work to reengineer the Mercury spacecraft would represent resources unavailable to develop Gemini, and secondly because of the questionable 


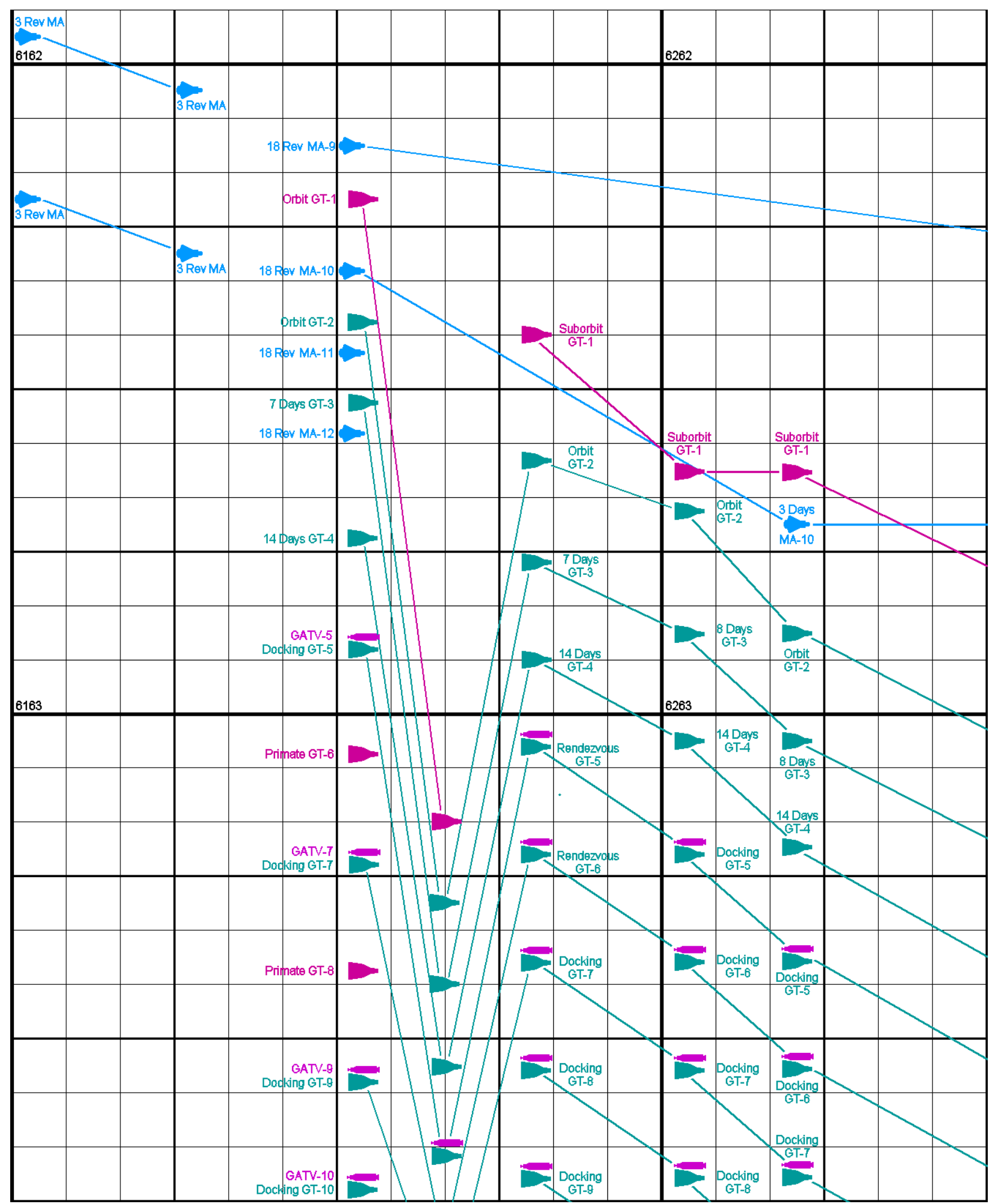

Figure 4. Time Map of Early US Manned Space Mission Planning (1 of 5) 


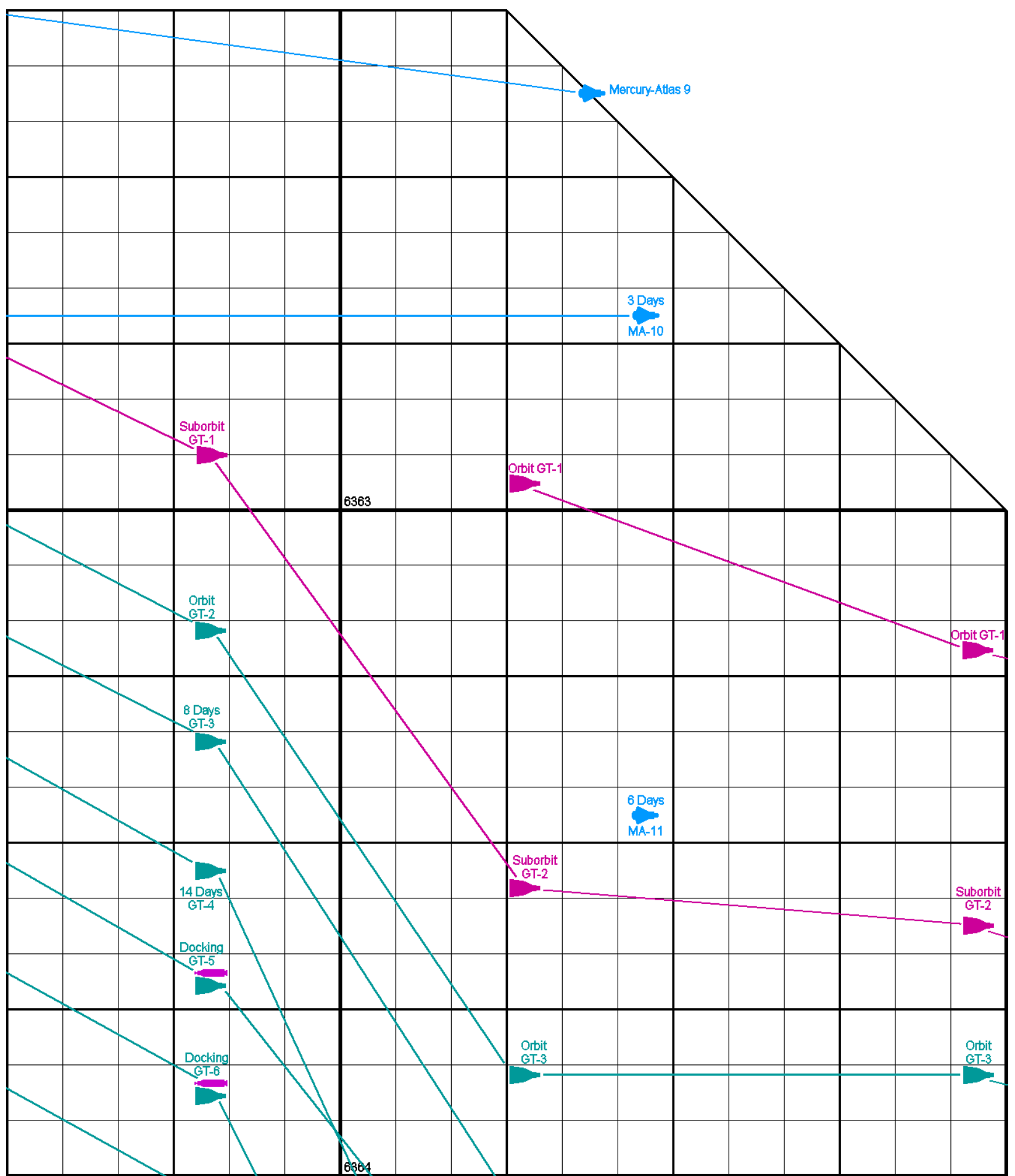

Figure 5. Time Map of Early US Manned Space Mission Planning (2 of 5)

wisdom of extending the capability of a very limited spacecraft that didn't have much of a future, given that a spacecraft designed with a 14-day capability would be flying only a few months later. The MA-9 mission probably wouldn't have survived, if not for the fact that the Gemini program suffered severe schedule slips early on. By March 1962, the first manned Gemini mission had slipped to November 1963, if MA-9 could be launched 10 months ahead of that, it might be worth it. By the time Walter Schirra flew his MA-8 mission in October 1962, the first manned Gemini mission had slipped to March 1964, which solidified MA-9 as both a schedule and 
technological gap-filler mission. Additionally, room was opening up in the schedule for a 3-day MA-10 mission, and even a 6-day MA-11 flight. However, a month after Cooper's MA-9 flight NASA terminated Project Mercury in favor of pushing ahead with Project Gemini without further distraction. Alan Shepard, who had rotated to the top of the flight roster, and now seeing his earliest opportunity for an orbital mission disappear in a programmatic decision, protested all the way to the White House. By then, the first manned Gemini mission, which presumably Shepard would command in lieu of flying MA-10, had slipped to November 1964, 14 months later than a possible MA-10 launch date.

Figures 6 through 8 show the bottom the Gemini program lineup as it appeared from 1961 through the end of 1963. Several things are interesting to note. In early program planning, missions were scheduled approximately two months apart, an aggressive schedule that had never been contemplated for the Mercury program. As the Gemini concept evolved from a scaled-up Mercury to a wholly new design with new technological challenges, the launch schedule slipped dramatically. By April 1963, management philosophy shifted from 2-month to 3-month launch centers, greatly expanding the program schedule. Whereas the 12-mission program settled on in October 1961 was then scheduled to conclude in March 1965, the final mission was now scheduled for January 1967.At this point, the program schedule began to solidify. Although some individual missions would slip a few months, and some would be substituted for others, overall, the program finished up slightly ahead of this April 1963 schedule, flying its final mission in November 1966.

Also to be seen in Figures 6 through 8 are layers made visible for other programs such as Dyna Soar, Manned Orbiting Laboratory, and Apollo. The time map display provides a wealth of information to the historical researcher and the program manager alike.

\section{Further Development}

At the present time, the slip chart appears little used. In nine years of space program management for the US Air Force, including attending some project management seminars, I never encountered it. A cursory search of the available software tools (Figures 9 through 11) revealed very limited capability, far from approaching the richness of features and detail that I have envisioned for time maps. The fact that the examples presented in these figures have the time axes in various orientations - a complete lack of standardization - is evidence of how these ideas have developed in isolation from each other and have failed to diffuse throughout the user community of project managers (see Figure 12).

An important dimension of project management is being overlooked. The credibility of a current project schedule to some degree derives from how successful the project has been at holding to its previous schedules. If the present schedule is the same one that has been used for some time, one has reason, based on history, to believe that the project will be able to hold to that schedule. But chances are that the schedule now being worked to is not the one that was published at the beginning of the program. It may not be the second, or even the third, if managing a high-risk, success-oriented program, and the technological breaks — or the money - haven't all gone the right way. If this is the case, how much faith can one place in the current schedule?

There is your program's Gantt chart, up there on the screen in front of you, as you sit in your weekly or monthly program review: the program schedule. How valid is it? The chart itself may give you some information. It may be a nice, clean series of horizontal bars with those progress bars keeping pace, or it may be that some tasks have extended to the right and the schedule has slipped. And if there are two or three extended tasks, how long have they been there.... month, a year? Is a trend developing that would indicate that other milestones are about to slip, or are you now successfully holding to a schedule that suffered some hemorrhaging six or seven months ago? Also, is this Gantt chart the one with which you began the program, or is it a re-baselined schedule from which earlier schedule slips have been erased? As you look up to the screen at the front of the conference room, the chart you see offers few clues to these questions. It depicts your current prediction of how the future is going to unfold, but it does not adequately tell the history of your concerted efforts to get to that future.

This is because that chart in front of you is really a series of charts, each of which has only one dimensiontime - running from left to right across the page. The vertical dimension is an illusion; it doesn't signify a continuum, but is simply a means of compiling the schedule of individual albeit interdependent milestones onto one visual display. It is today's snapshot of how those milestones relate to each other in the overall schedule, but it doesn't show all that well how those milestones have influenced each other over time. Wouldn't it be valuable to have some way of displaying all of yesterday's schedule snapshots as well as today's on a single chart? Then you could see at a glance the nature of the trends for each milestone and how they feed into the trend of the master schedule. 


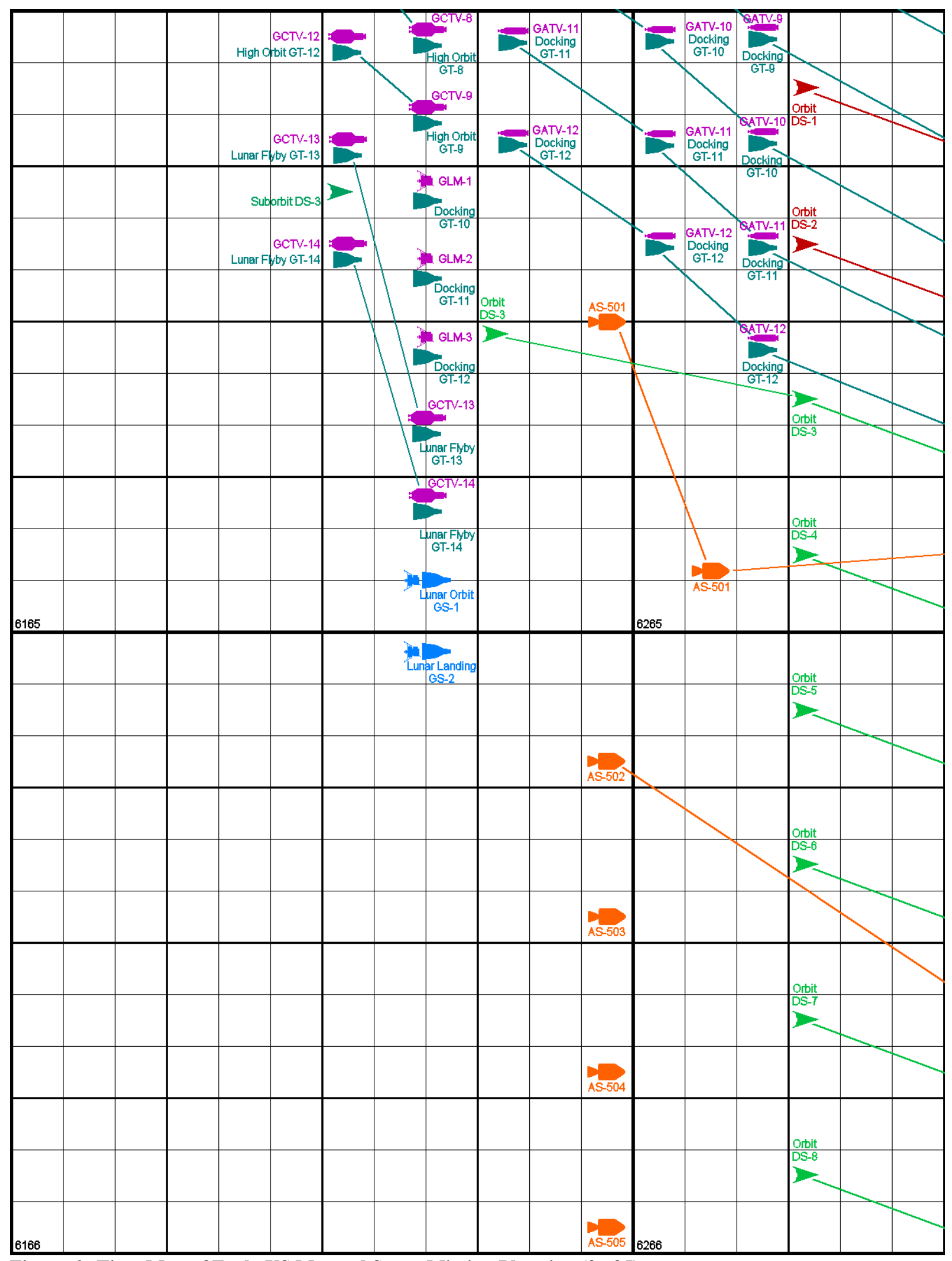

Figure 6. Time Map of Early US Manned Space Mission Planning (3 of 5) 


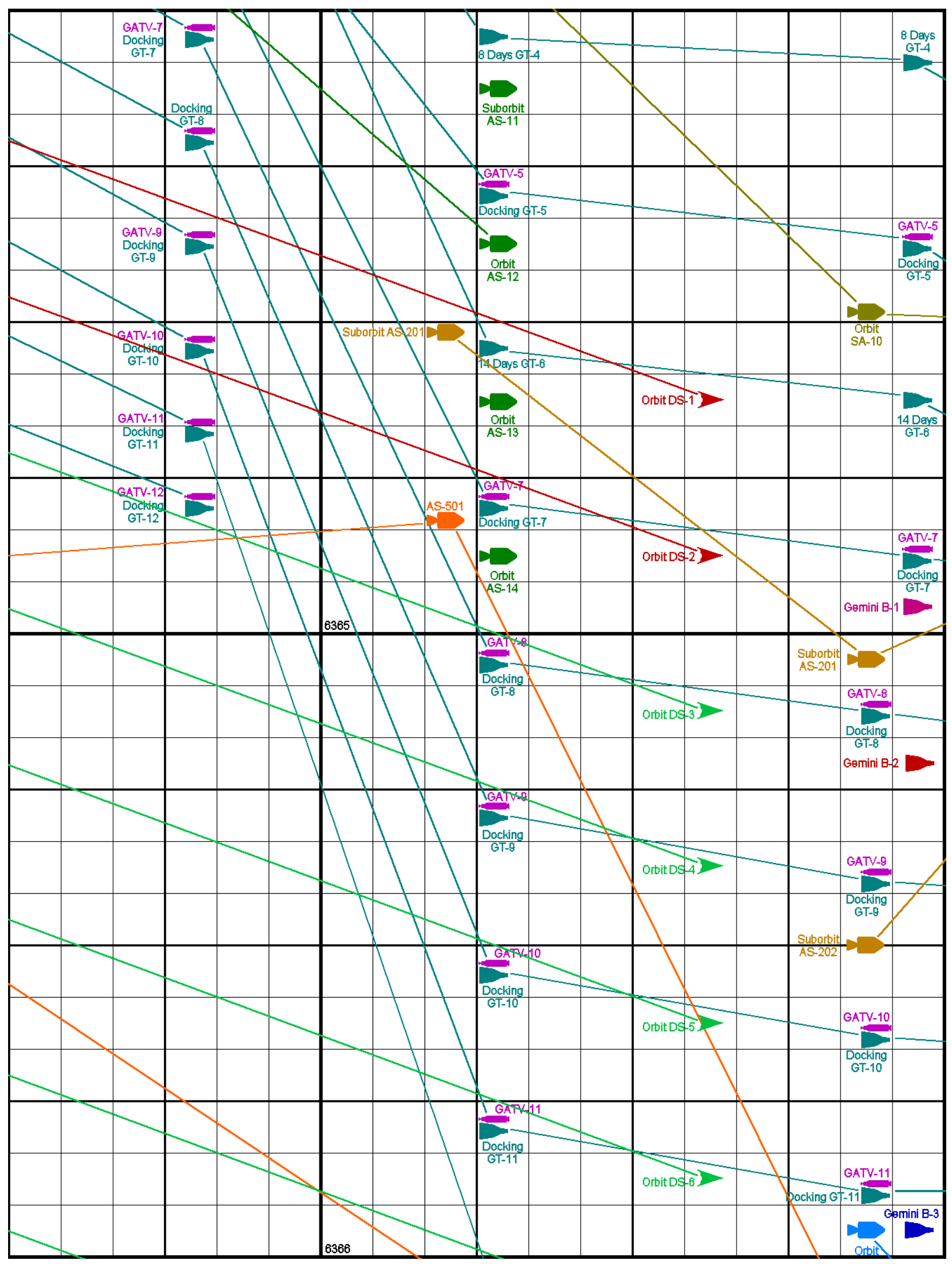

Figure 7. Time Map of Early US Manned Space Mission Planning (4 of 5) 


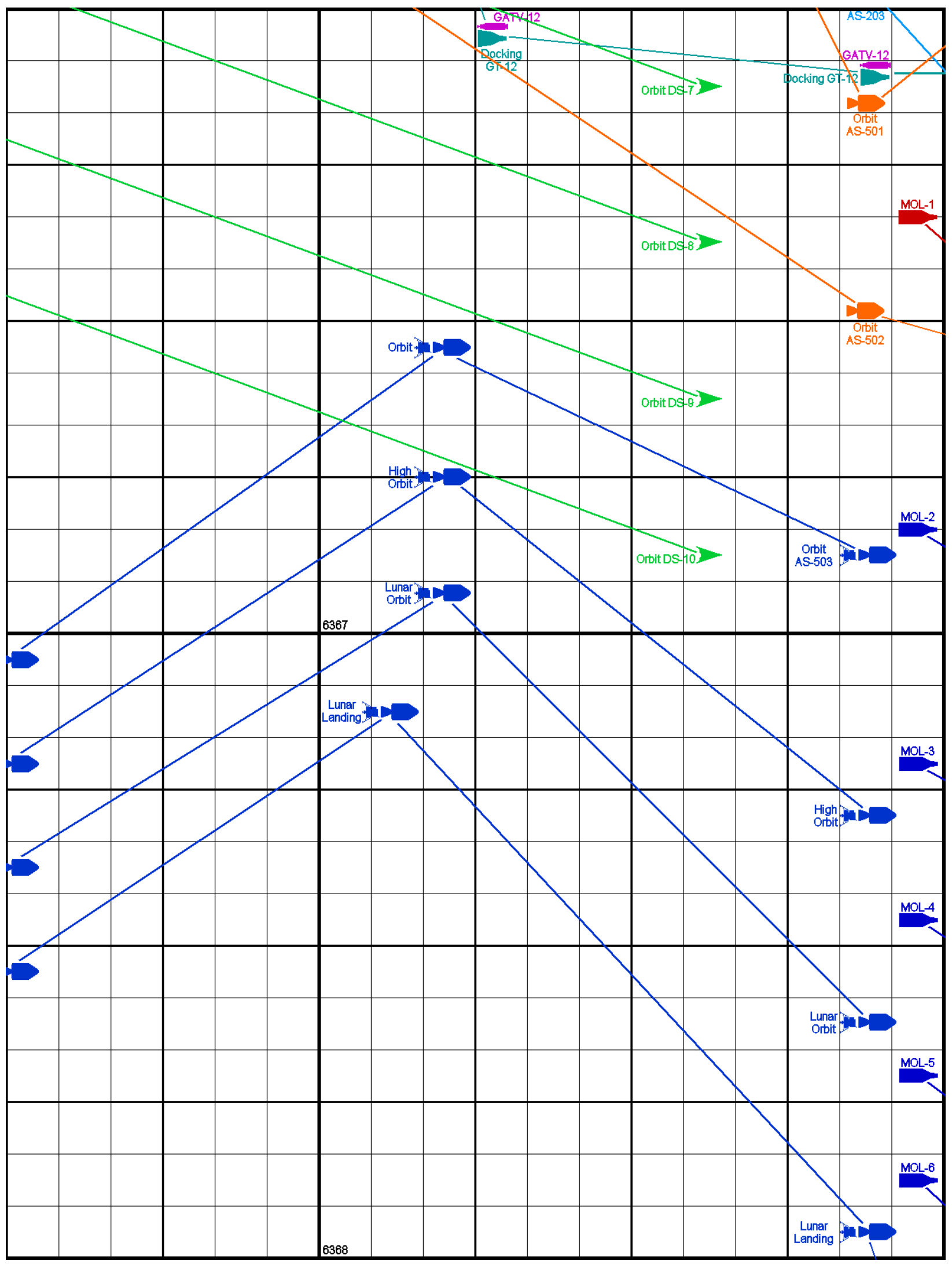

Figure 8. Time Map of Early US Manned Space Mission Planning (5 of 5) 


\section{PD-Trak Slip Chart}

Virgo

Slp is the difference between the remaining development tme based on the current plan and the development time as defined by the baseine plan. The cabculation is based on the predicted time between the folowing gates:

CD Gates ... to .... eReview?

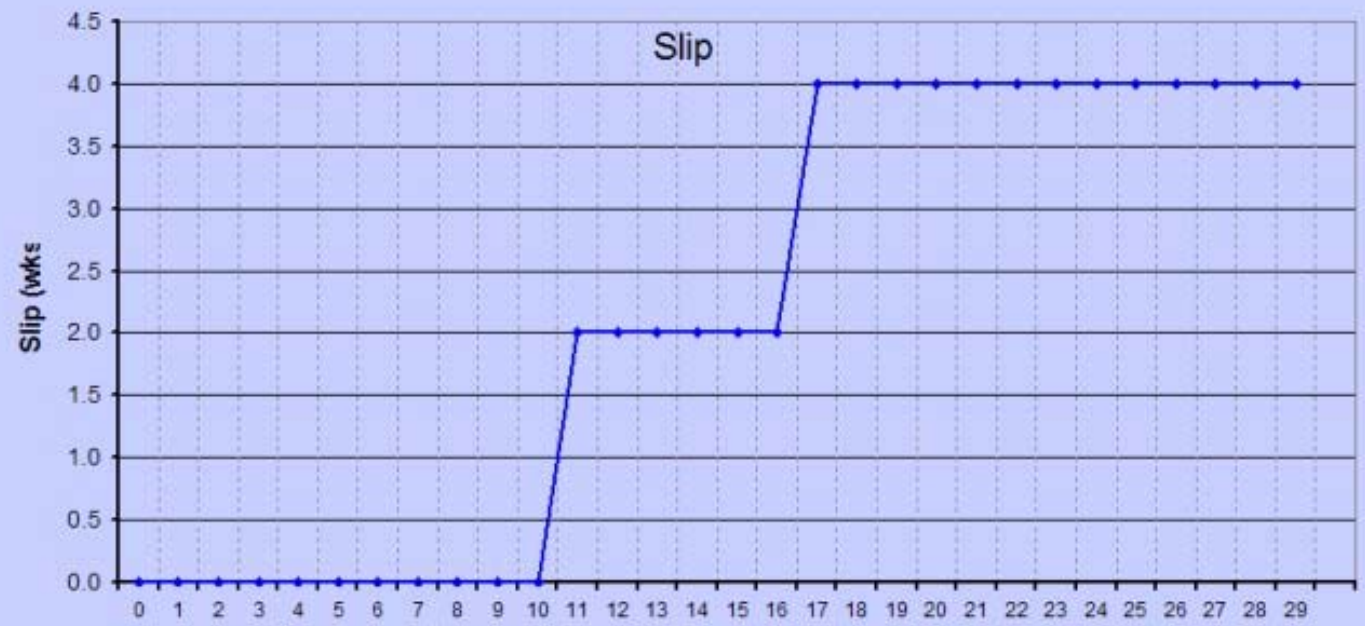

Figure 9. PD-Trak Slip Chart

Source: http://www.pd-trak.com/perfmeas.htm

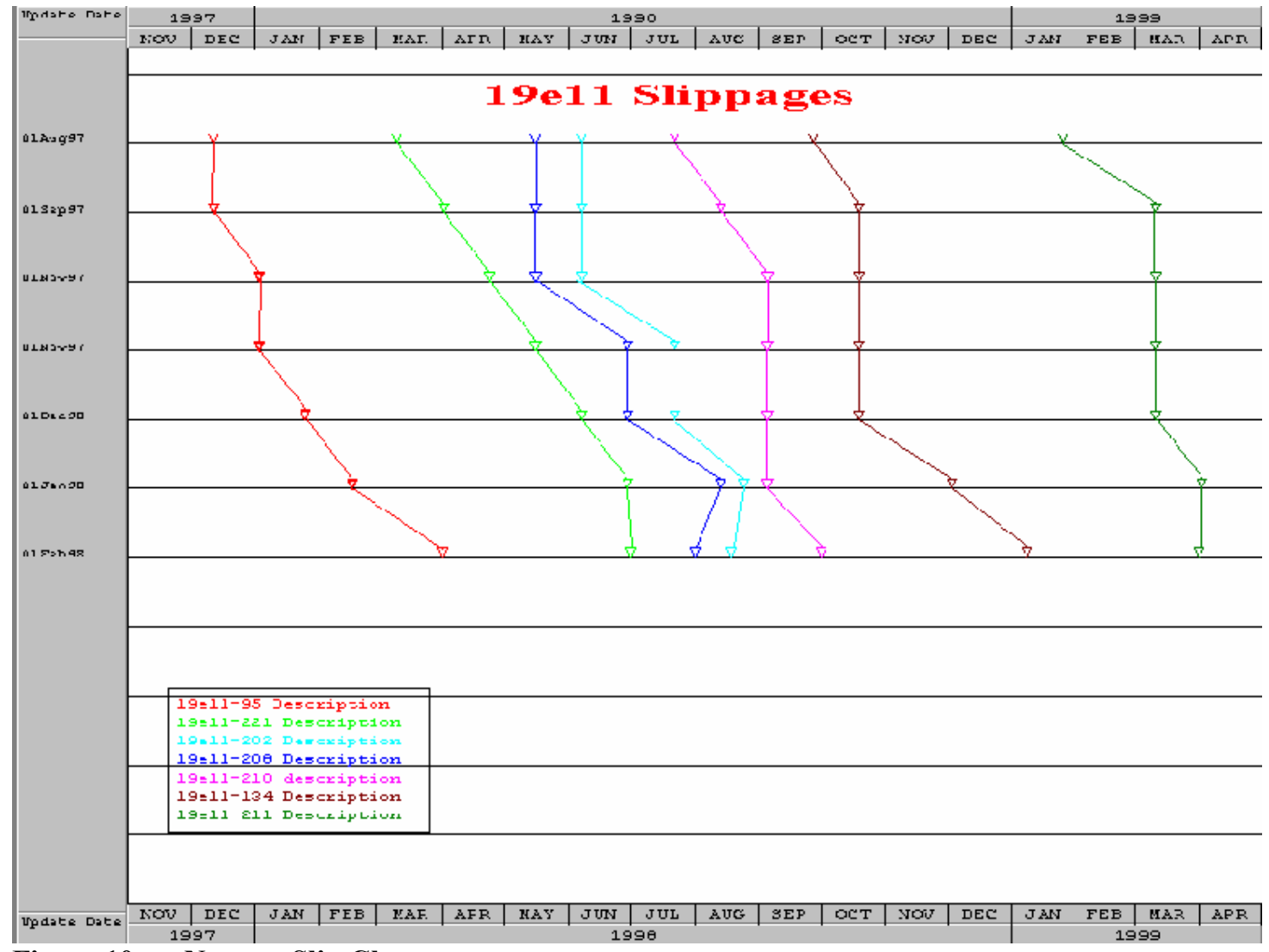

Figure 10. Noweco Slip Chart

Source: http://www.noweco.com/qei/qeie10.htm 


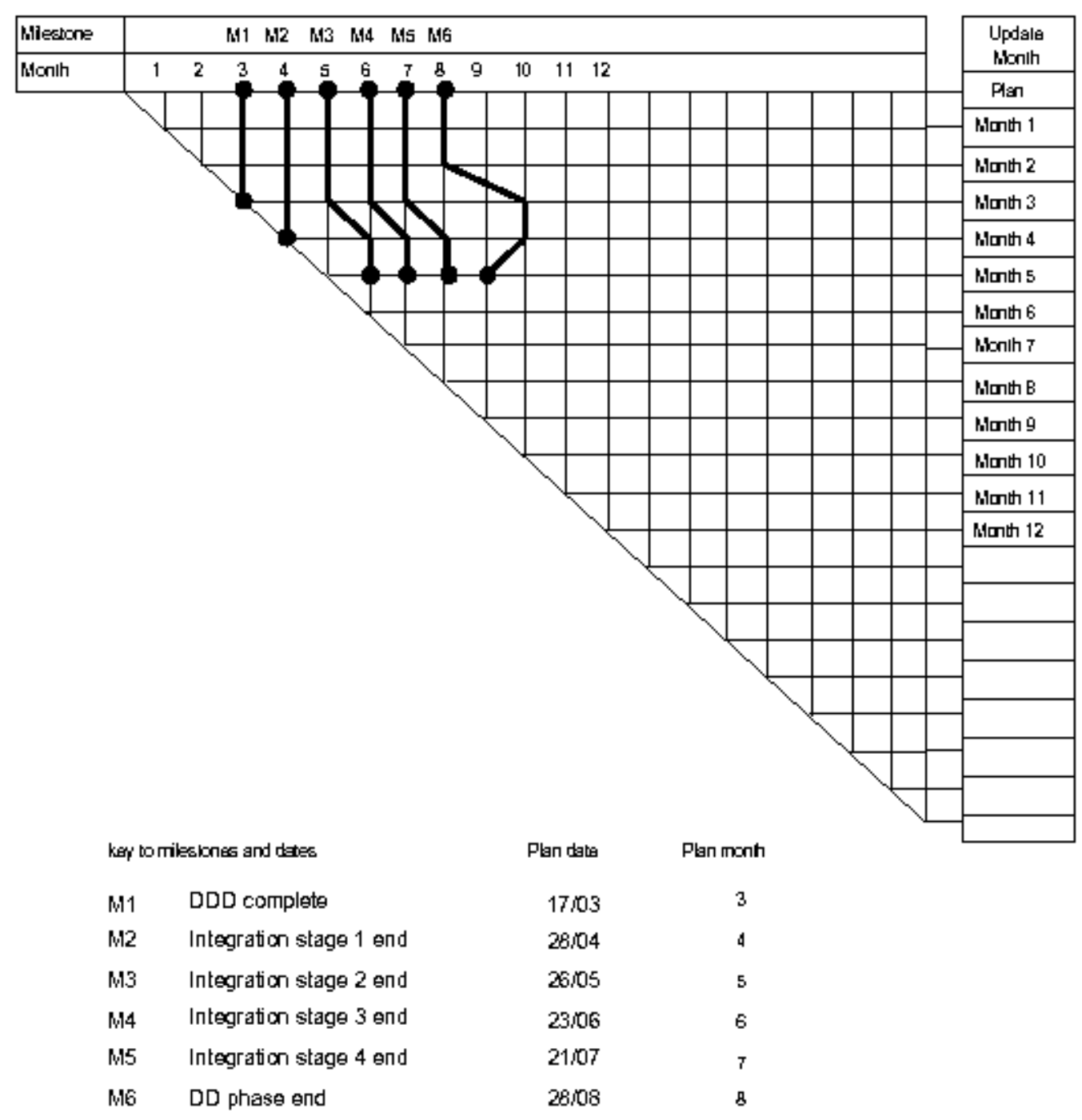

Figure 11. ESA Slip Chart

Source: http://styx.esrin.esa.it/premfire/Docs/PSS0508.pdf

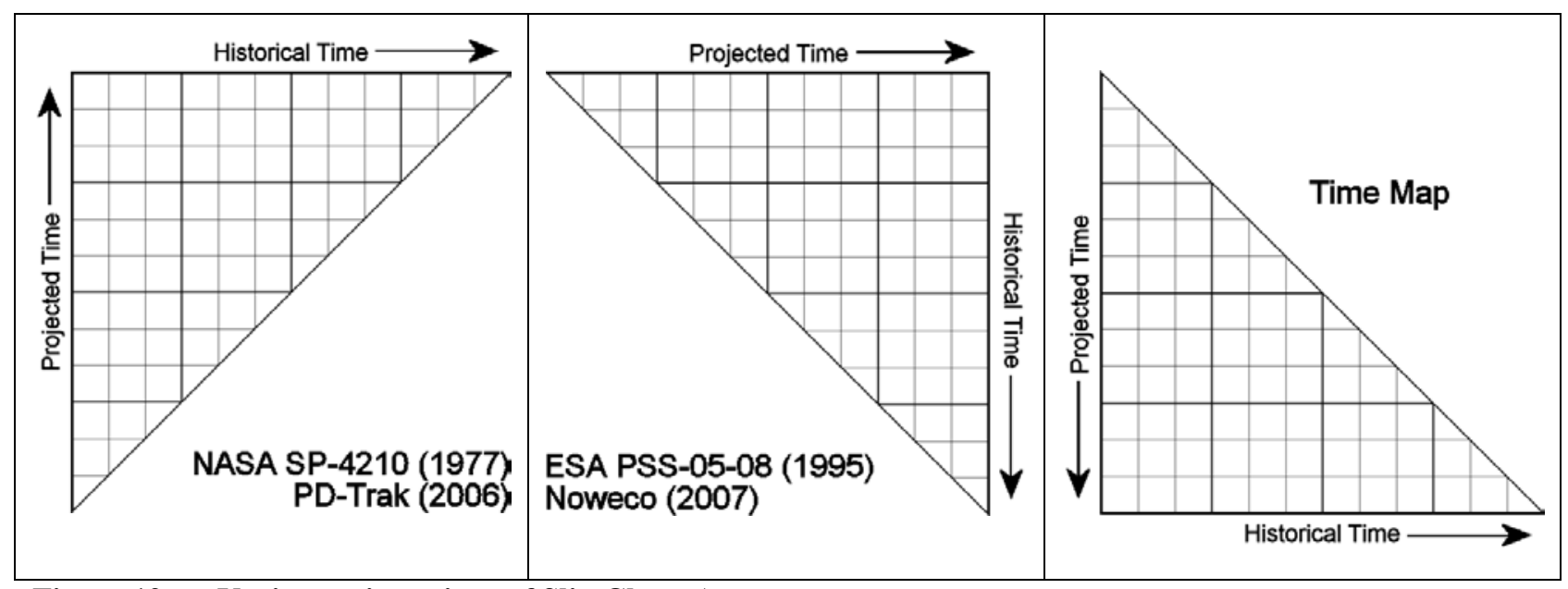

Figure 12. Various orientations of Slip Chart Axes 
This can be done by reformatting the chart so that a second dimension of time is displayed. After all, the time axis on a conventional schedule chart isn't real time at all, not in the sense of showing the occurrence of actual events with respect to the passage of time; it is only projected time - the pseudo-temporal axis along which a manager positions his best guesses of how he will achieve his milestones. When he does indeed achieve those milestones, only then do they become historical events and fall into the domain of the real passage of time. In a chart that not only contains the pseudo-temporal axis but also a second axis corresponding to the actual passage of time, one can see the history of a program's perception of the future - how schedules have been influenced by the course of events, and how they have evolved with time (Figure 3). In short, you will be able to see at a glance not only where you are now and where you plan to be when, but also how you got to where you are now. Presentation of schedules in this new two-dimensional format provides managers an extra dimension of information, enabling them to make better subjective judgments on how realistic the current schedule is, and how it may change in the face of possible future events.

\section{Analytic Tools}

Figure 13 is a time map of a simplified program. At this point, several general observations can be made about time maps. First of all, the orientation of axes is projected time from top to bottom and historical time from left to right. This orientation is in keeping with the orientation of the Gantt chart, in which time is read from left to right, and earlier tasks tend to appear at the top and latter tasks at the bottom. Next, notice that all completed milestones fall on a diagonal line from upper left to lower right. This line is the "event horizon"-where schedule becomes reality, where the future becomes history. The event horizon is one of the lines that defines the characteristic shape of the time map. Another is the vertical line on the far left that is defined by the first schedule of the program. At the bottom of the time map is the third boundary, which is defined by the date of the latest milestone that was scheduled in the program. Lastly, in the lower left corner of the time map is the "depth of schedule", the diagonal line that parallels the event horizon and is marked by the milestone that was scheduled most in advance of its intended date of completion.

The time map can be studied from the perspective of either axis. For instance, at any point in historical time, i.e., a fixed point on the horizontal axis, the then existing program schedule can be seen along the vertical axis into the future. Or, following the historical perspective, the scheduling history of any specific milestone can be traced from beginning to end.

Perhaps the most valuable aspect of the time map is that it makes possible two new and quantitative management tools. The first of these is the concept of schedule slope, which is defined as the ratio of the change in schedule and the passage of historical time, or:

$$
s=\frac{t_{p}}{t_{h}}=\frac{t_{p 2}-t_{p 1}}{t_{h 2}-t_{h 1}}=\frac{\text { change in projected time }}{\text { change in historical time }}
$$

Being the ratio of two time quantities, $s$ is dimensionless. The quantity $t_{h}$ is always a positive value, of coursehistorical time doesn't run backward. On the other hand, $t_{p}$ can either be a positive, negative, or zero quantity. A positive value for $t_{p}$ occurs when $t_{p 1}$ is greater than $t_{p 2}$, or in other words when a milestone appears earlier on a subsequent schedule than it does on a previous one. This results in a positive slope, which signifies schedule gain. When $t_{p l}=t_{p 2}$, then $t_{p}=0, s=0$, and the program is proceeding right on track. When $t_{p 1}$ is less than $t_{p 2}$, then $t_{p}$ is less than zero and the slope is negative, representing schedule loss. A schedule slope of -1 means that the milestone is in a one-for-one slip, i.e., day-for-day or month-for-month, and essentially no progress is being made toward its accomplishment.

A second quantity that can be derived from the time map is one which expresses the rate of relative expansion or compression of schedule between two milestones over the passage of historical time. This concept can be thought of as the expansion rate of a schedule:

$$
e=\frac{\frac{\left(t_{p 2 B}-t_{p 2 A}\right)-\left(t_{p 1 B}-t_{p 1 A}\right)}{t_{p 1 B}-t_{p 1 A}}}{t_{h 2}-t_{h 1}}
$$




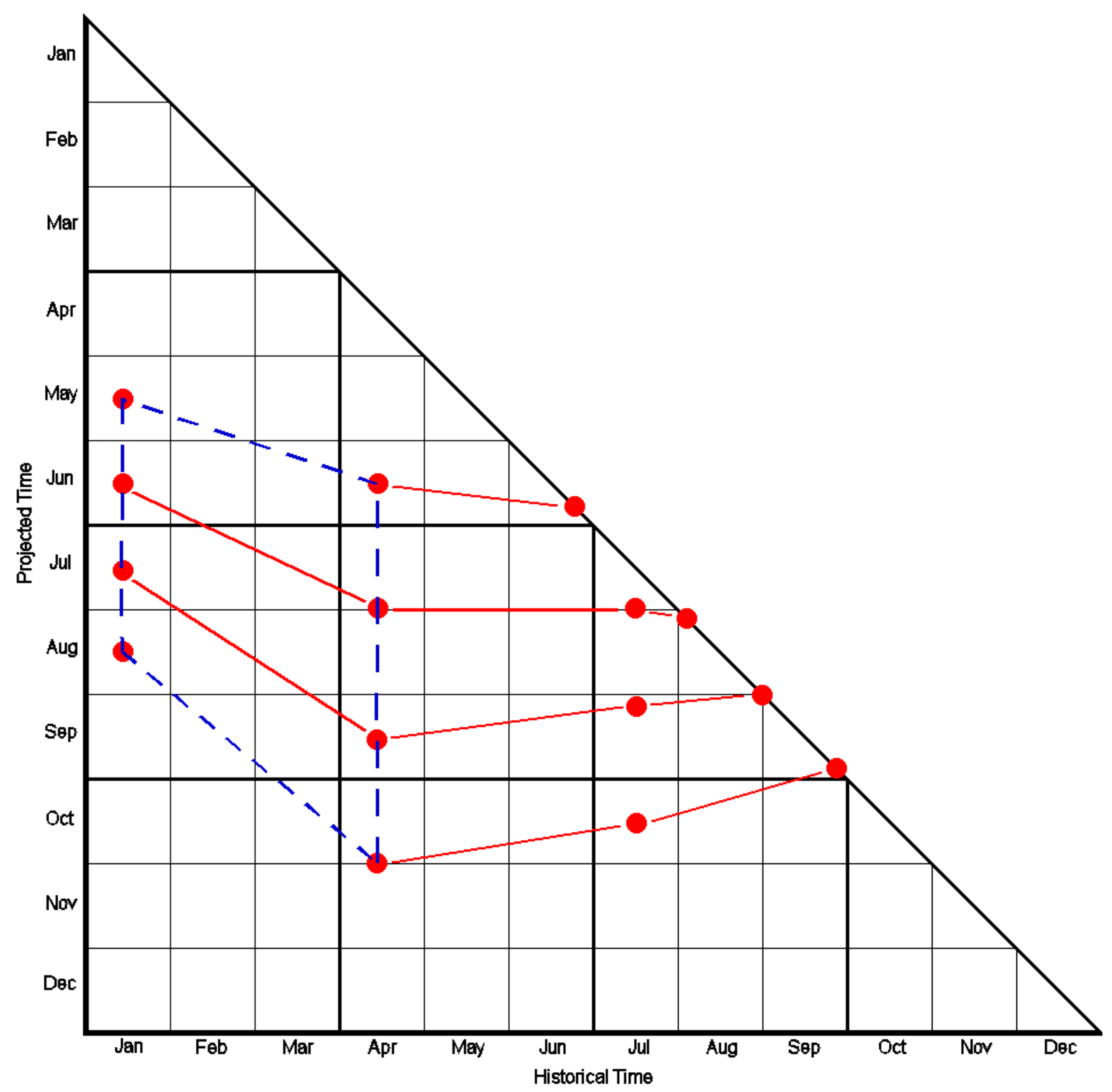

Figure 13. Hypothetical Development Program

When $e$ is positive, the schedule is expanding as indicated by the dashed outline in Figure 13. In contrast, a negative value of $e$ signifies compression of schedule. The larger the proportional difference between the vertical length of the two schedules being compared, the larger the magnitude (either positive or negative) of $e$.

Keeping the proportionality of the two schedules constant, the greater the length of historical time between the two schedules, the smaller the magnitude of $e$.

\section{Software Capabilities}

Color and symbols can be used to differentiate between various types of milestones, i.e., final assembly, systems test, delivery, operational status, et cetera. Or in cases where it is useful to simultaneously display the schedules of several different programs, each program can be represented by a different symbol. For instance, several programs may share the use of the same support facilities, and the schedule aspects of these programs that are pertinent to facilities scheduling may be displayed on a single chart using different symbols. It should also be noted that, just as 
the conventional map representing two dimensions of space can depict the layout of a village or the conformation of continents, so the time map can be expanded or condensed to any desired time scale.

\section{Conclusion}

The conventional Gantt schedule chart is designed primarily for the purpose of displaying the current planning of activities on a program. It is not well suited to depicting the ebb and flow of events in the life of a program as a variety of forces influence the project over time. In reality, such a chart contains only one dimension of time: that momentary vision of the future which exists in the collective mind of the program management. By adding a dimension - that of historical time - the evolution of management's view of the future can be displayed. Furthermore, in this new two-dimensional environment, important trend information becomes visible and quantifiable for the first time, enabling managers to make better-informed judgments concerning the credibility of the current program schedule.

The two-dimensional time format liberates us from the conventional perception of time as being linear and unidirectional, and allows us to see time in its two distinct components. One axis of time is the past - the domain of deeds already done. The other element of time is the future, which exists in the separate dimension of human imagination and planning. Together they form the surface of time, and it is only on this two-dimensional surface that our journey between the past and the future can be properly mapped out.

\section{References}

Brooke, D. G. 1973. "The Use of Slip Charts to Review Research Projects," RD Management, Vol. 4 Issue 1 Page 9. October. Internet. Available from http://www.blackwell-synergy.com/doi/abs/10.1111/j.1467-9310.1973.tb01026.x?journalCode =radm; accessed 5 August 1007 .

European Space Agency. 1995. Guide to Software Project Management. PSS-05-08 Issue 1 Revision 1 March 1995. Internet. Available from http://styx.esrin.esa.it/premfire/Docs/PSS0508.pdf; accessed 5 August 2007.

Gantt, H. L. 1910. "Work, Wages and Profit." The Engineering Magazine. New York. Republished as Gantt, H. L. (1974). Work, Wages, and Profits. Easton, Pennsylvania: Hive Pub. Co.

Hall, R. Cargill. 1977. Lunar Impact-A History of Project Ranger. NASA 1977 SP-4210. Internet. Available from http://history.nasa.gov/SP-4210/pages/Cover.htm; accessed 5 August 2007. 